\title{
EVIDENCE FOR MULTIPLY CHARGED ANOMALOUS COSMIC RAYS
}

\author{
R. A. Mewaldt, R. S. Selesnick, J. R. Cummings, and E. C. Stone \\ California Institute of Technology, Pasadena, CA 91125
}

AND

T. T. von RosENVINGE

Goddard Space Flight Center, Code 661, Greenbelt, MD 20771

Received 1996 March 27; accepted 1996 May 14

\begin{abstract}
New measurements from the $S A M P E X$ spacecraft show that most anomalous cosmic ray (ACR) oxygen nuclei with energies above $20 \mathrm{MeV}$ nucleon ${ }^{-1}$ are multiply ionized, with ionic charge states of $Q=2, Q=3$, and probably higher. This new result contrasts with lower energies, at which most ACRs are singly charged. The observed abundance of multiply charged ions agrees with estimates of the fraction of singly charged ACRs that undergo electron stripping during acceleration if the timescale for acceleration to $10 \mathrm{MeV}$ nucleon $^{-1}$ is $\sim 1 \mathrm{yr}$ The existence of multiply charged ACRs helps explain their acceleration to high energies, and it has implications for several other studies.

Subject headings: cosmic rays — interplanetary medium — solar wind
\end{abstract}

\section{INTRODUCTION}

During the early 1970s, a new energetic particle component was discovered when anomalous enhancements were observed in the quiet-time energy spectra of $\mathrm{He}, \mathrm{N}, \mathrm{O}$, and $\mathrm{Ne}$ with 5-50 $\mathrm{MeV}_{\text {nucleon }}^{-1}$ (see review in Klecker 1995). The accepted explanation for these anomalous cosmic rays (ACRs) (Fisk, Kozlovsky, \& Ramaty 1974) is that they originate from interstellar neutral atoms that have been swept into the heliosphere and ionized by solar UV or charge exchange with the solar wind to become solar wind pickup ions. The pickup ions are then convected into the outer heliosphere and accelerated to tens of $\mathrm{MeV}$ per nucleon, probably at the solar wind termination shock (Pesses, Jokipii, \& Eichler 1981).

A fundamental prediction of the above theory is that ACRs should be singly charged, unlike Galactic cosmic rays (GCRs) or solar energetic particles. There is abundant evidence that the bulk of ACRs with $\sim 10 \mathrm{MeV}$ nucleon $^{-1}$ have an ionic charge state of $Q=1$. Following several indirect tests of this prediction (e.g., Klecker et al. 1980; Cummings, Stone, \& Webber 1984), Adams et al. (1991) found $Q=0.9_{-0.2}^{+0.3}$ for 5-11 MeV nucleon ${ }^{-1}$ ACR oxygen by comparing orbit-averaged fluxes inside the magnetosphere with interplanetary fluxes. A more direct approach traces individual particle trajectories back through the geomagnetic field to place limits on the charge state (Oschlies, Beaujean, \& Enge 1989; Singh et al. 1991), and recently, Klecker et al. (1995), using the Heavy Ion Large Telescope sensor on $S A M P E X$, found that more than $90 \%$ of $8-16 \mathrm{MeV}$ nucleon ${ }^{-1}$ ACR oxygen is singly charged.

Another SAMPEX study (Mewaldt et al. 1996) used Earth's field to filter ACRs from GCRs and found that the spectra of ACR N, O, and $\mathrm{Ne}$ all extend to above $70 \mathrm{MeV}$ nucleon $^{-1}$, several times the characteristic "maximum" energy expected for diffusive shock-drift acceleration at the termination shock (Jokipii 1990). To explain how ACRs could attain such high energies, Mewaldt et al. (1996) suggested that the highest energy ACRs may be multiply charged, in which case this acceleration process achieves greater energy per nucleon. In this Letter, we test the hypothesis that the highest energy ACRs have $Q>1$.

\section{OBSERVATIONS AND INTERPRETATION}

The observations were made with the Mass Spectrometer Telescope (MAST) on the polar-orbiting SAMPEX satellite from 1992 July 7 to 1995 February 18. MAST measures the charge, mass, kinetic energy, and trajectory of incident ions, including oxygen from $\sim 16$ to $\sim 160 \mathrm{MeV}$ nucleon $^{-1}$. The measured kinetic energy versus invariant latitude $(\lambda)$ for quiet-time oxygen events is shown in Figure 1, based on 865 days of data with daily average $8-15 \mathrm{MeV}^{\text {nucleon }}{ }^{-1}$ helium fluxes of less than $4\left(\mathrm{~m}^{2} \mathrm{sr} \mathrm{s}\right)^{-1}$. Invariant latitude is defined by $\cos ^{2} \lambda=1 / L$, where $L$ is the McIlwain $L$-parameter (see, e.g., Roederer 1970). The approximate geomagnetic cutoffs for vertically incident $\mathrm{O}^{+}, \mathrm{O}^{+2}, \mathrm{O}^{+3}, \mathrm{O}^{+4}$, and $\mathrm{O}^{+8}$, shown in Figure 1, are based on a modified Størmer dipole theory, described below.

Previous $S A M P E X$ studies identified a population of $\mathrm{N}, \mathrm{O}$, and $\mathrm{Ne}$ ions near $\lambda=45^{\circ}$ originating from ACRs that have become trapped in the inner radiation belt. Selesnick et al. (1995) found that trapped ACRs with $16-50 \mathrm{MeV}^{\text {nucleon }}{ }^{-1}$ have pitch angles of $60^{\circ}-120^{\circ}$ and are bounded in latitude by the requirement for adiabatic motion, characterized by $\epsilon Q<1$ (dashed line in Fig. 1). Here $\epsilon=3 \mathscr{R} /\left(L R_{\mathrm{E}} B_{0}\right)$, where $\mathscr{R}$ is magnetic rigidity, $R_{\mathrm{E}}$ is Earth's radius, and $B_{0}$ is the equatorial magnetic field strength. Using these properties, we have excluded trapped ACRs from Figure 1 and this analysis.

From the observation of a single particle at a given latitude, it is possible only to determine an upper limit on its charge state because lower charge state particles also have access to that latitude. However, it is also possible to estimate the distribution of charge states in a particle population from their latitude distribution. The extent to which ACRs penetrate the geomagnetic field is evident in Figure 1. At low energies $\left(E<30 \mathrm{MeV}\right.$ nucleon $\left.^{-1}\right), \mathrm{ACR}$ oxygen is observed at latitudes down to the $\mathrm{O}^{+}$cutoff. However, above $30 \mathrm{MeV}$ nucleon $^{-1}$ there are only three events observed at latitudes 


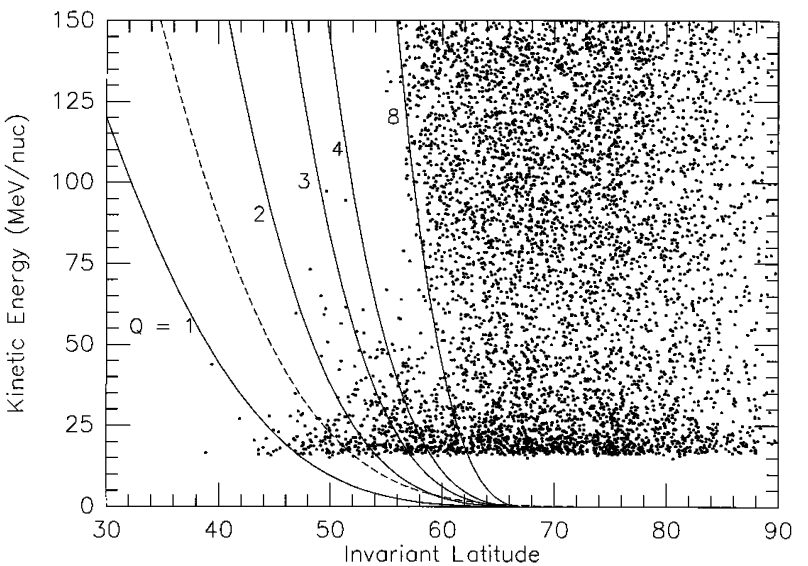

FIG. 1.-Measured energy per nucleon vs. invariant latitude for quiet-time oxygen events. Also shown are calculated vertical geomagnetic cutoffs for $\mathrm{O}^{+}, \mathrm{O}^{+2}, \mathrm{O}^{+3}, \mathrm{O}^{+4}$, and $\mathrm{O}^{+8}$ and the upper boundary for trapped ACRs (dashed line).

below the $\mathrm{O}^{+2}$ cutoff, compared to more than 30 between the $\mathrm{O}^{+2}$ and $\mathrm{O}^{+3}$ cutoffs.

Oxygen latitude distributions are shown in four energy intervals in Figure 2. To determine the distributions expected from a given charge state, we have used the Størmer theory for the cutoff in a dipole magnetic field with some empirical adjustments. A particle is assumed to have access if its rigidity is greater than the cutoff rigidity, given by

$$
\mathscr{R}_{c}=\frac{C_{s}\left(\cos ^{4} \Lambda-\cos ^{4} \Lambda_{o}\right)}{R^{2}\left[1+\left(1-\sin \theta \cos \phi \cos ^{3} \Lambda\right)^{1 / 2}\right]^{2}} .
$$

Here $C_{s}=57.9 \mathrm{MV}$ is Earth's dipole moment converted to a rigidity, $\theta$ and $\phi$ are the trajectory zenith and azimuth angles, respectively (azimuth measured from east to north), and $\Lambda$ and $R$ are the magnetic latitude and equivalent radius from the dipole center, respectively (see, e.g., Roederer 1970). To account for the open polar cap, $\mathscr{R}_{c}$ includes an empirical offset, $\Lambda_{o}=67^{\circ}+D_{\text {st }} / 16.5$ (where $D_{\text {st }}$ is a geomagnetic activity index), derived from solar particle observations (Leske et al. 1995). Included in Figure 2 are fits to the latitude distributions for (1) a mixture of only $\mathrm{O}^{+8}$ GCRs and $\mathrm{O}^{+}$ACRs and (2) a mixture of $\mathrm{O}^{+}, \mathrm{O}^{+2}, \mathrm{O}^{+3}$, and $\mathrm{O}^{+8}$. In each energy interval, the charge state abundances were varied to obtain the best fit to all latitude bins above the $\mathrm{O}^{+}$cutoff. It is clear that singly charged ACRs alone cannot account for the observations, particularly above $30 \mathrm{MeV}$ nucleon ${ }^{-1}$. Table 1 summarizes the reduced $\chi^{2}$ for fits with maximum ACR charge states ranging from $\mathrm{O}^{+}$to $\mathrm{O}^{+4}$. In all but the lowest energy interval, the fits are significantly better when $\mathrm{O}^{+2}$ and $\mathrm{O}^{+3}$ are included. From 40 to $54 \mathrm{MeV}$ nucleon ${ }^{-1}, \mathrm{O}^{+3}$ is found to be the most
TABLE 1

Goodness of Charge State Fits

\begin{tabular}{ccccc}
\hline \hline $\begin{array}{c}\text { INCLUDED ChARGE } \\
\text { STATES }\end{array}$ & \multicolumn{3}{c}{ REDUCED $\chi^{2}\left(\mathrm{MeV}\right.$ nucleon ${ }^{-1}$ bins $)$} \\
\cline { 2 - 5 } & $16-22$ & $22-30$ & $30-40$ & $40-54$ \\
\hline $1,8 \ldots \ldots \ldots \ldots \ldots \ldots \ldots$. & 1.79 & 1.66 & 3.38 & 2.45 \\
$1,2,8 \ldots \ldots \ldots \ldots \ldots \ldots$. & 1.87 & 0.83 & 0.93 & 1.35 \\
$1,2,3,8 \ldots \ldots \ldots \ldots \ldots$. & 1.89 & 0.80 & 0.79 & 0.99 \\
$1,2,3,4,8 \ldots \ldots \ldots \ldots$. & 1.99 & 0.84 & 0.82 & 1.03 \\
\hline
\end{tabular}

abundant charge state, consistent with the improvement in the reduced $\chi^{2}$ in Table 1 . The need for $\mathrm{O}^{+4}$ in addition to $\mathrm{O}^{+2}$ and $\mathrm{O}^{+3}$ is not established.

These results were checked with a Monte Carlo approach in which particle trajectories were traced backward by numerical integration through a geomagnetic field model that combined the 1992 International Geomagnetic Reference Field internal field with the Tsyganenko (1989) external field, modified to account for variations in geomagnetic activity (Boberg et al. 1995). Although this approach yields cutoffs $\sim 2^{\circ}$ higher than the modified Størmer cutoffs, the resulting fraction of multiply charged ACRs is similar. We present here the results of the Størmer approach, which agrees better with the observed GCR cutoffs and yields better overall fits.

Because the relative charge state abundances are subject to uncertainties in the geomagnetic cutoffs, we have combined the best-fit abundances of all $Q>1$ species into a single quantity. The resulting energy spectra of $Q=1$ and $Q>1$ ACRs, along with their sum, are shown in Figure 3. Integrating these, we find that multiply charged ions account for $\sim 50 \%-$ $60 \%$ of ACRs above $16 \mathrm{MeV}$ nucleon $^{-1}$ and more than $95 \%$ of ACRs with energies greater than $30 \mathrm{MeV}$ nucleon ${ }^{-1}$. Extrapolating to lower energies using typical ACR spectral shapes (see below), we estimate that at 1 AU multiply charged ACRs constitute $\sim 20 \%$ of the integral flux of ACRs above $1 \mathrm{MeV}$ nucleon $^{-1}$.

\section{DISCUSSION}

It may seem surprising that such a large fraction of highenergy ACRs are multiply charged in light of their origin as pickup ions and the previous verifications that most ACRs are singly charged. However, these previous studies were either at lower energy or were not sufficiently precise to identify multiply charged ACRs. The presence of multiply charged ACRs implies either that the source population contains a large fraction of multiply charged pickup ions or that many ACRs are stripped of additional electrons during their acceleration. The first possibility is unlikely because the abundance of pickup $\mathrm{He}^{+2}$ is only $\sim 2 \%$ of $\mathrm{He}^{+}$, with the abundance of

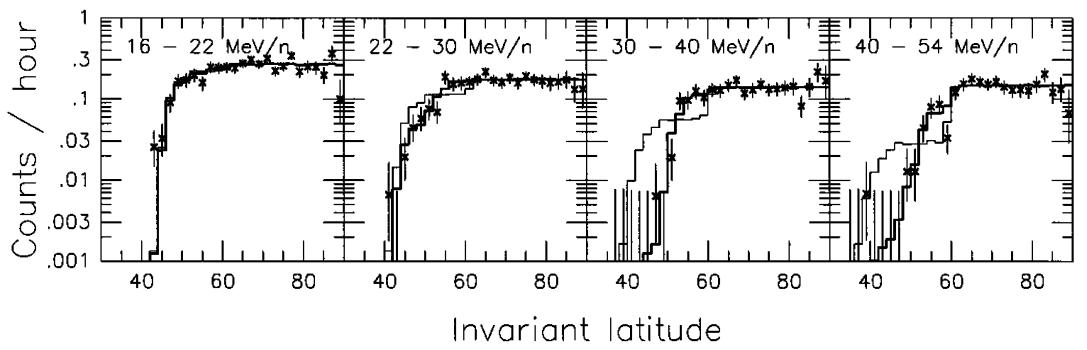

FIG. 2.- Measured latitude distributions of oxygen events in four energy intervals, along with fits to the distributions including charge states of $Q=1$ and 8 (light traces) and $Q=1,2,3$, and 8 (dark traces). 


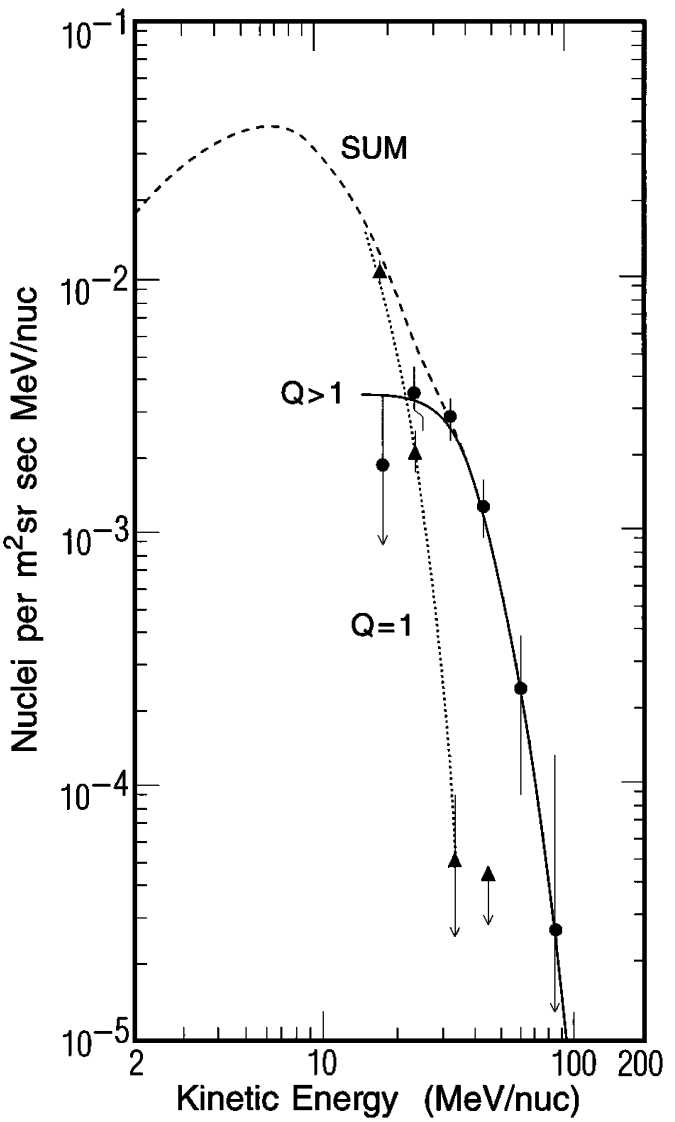

FIG. 3.-Measured energy spectra of singly and multiply charged ACRs, along with their sum (dashed line). The $O>1$ spectrum is the sum of the best-fit abundances of $\mathrm{O}^{+2}, \mathrm{O}^{+3}$, and $\mathrm{O}^{+4}$. Smooth lines have been drawn through the points to guide the eye. Below $16 \mathrm{MeV}$ nucleon $^{-1}$, the summed spectrum was estimated from other measurements (Mewaldt et al. 1993; Hasebe et al. 1994; Cummings et al. 1995).

multiply charged pickup oxygen expected to be much less (Geiss, Gloeckler, \& von Steiger 1995).

To evaluate electron stripping during ACR acceleration, we use the model of Jokipii (1992), who estimated a minimum time of $\sim 0.5 \mathrm{yr}$ to accelerate $\mathrm{O}^{+}$to $10 \mathrm{MeV}$ nucleon ${ }^{-1}$ at the termination shock (see also Klecker 1995). For a mean free path that is independent of rigidity, a reasonable approximation below 1 GV (Palmer 1982), the acceleration time is proportional to rigidity. Using cross sections for stripping $\mathrm{O}^{+}$ (Spjeldvik 1979) in an assumed neutral $\mathrm{H}$ density of $0.1 \mathrm{~cm}^{-3}$, we have evaluated the fraction of ions stripped to various charge states. These fractions are shown in Figure 4 for an acceleration rate that achieves $10 \mathrm{MeV}$ nucleon ${ }^{-1}$ in $1 \mathrm{yr}$. Note that, by $15 \mathrm{MeV}$ nucleon ${ }^{-1}, \sim 30 \%$ of the $\mathrm{O}^{+}$is stripped to $\mathrm{O}^{+2}, \mathrm{O}^{+3}$, or $\mathrm{O}^{+4}$, with most of the stripping occurring at energies of $\sim 0.1-1 \mathrm{MeV}$ nucleon $^{-1}$, where the cross sections are largest. It had previously been assumed that most stripping occurs at higher energies (Jokipii 1992; Klecker 1995). Similar results, with somewhat less stripping for a 1 yr timescale, are obtained for an acceleration time proportional to energy. Slower acceleration rates produce proportionally more multiply charged ACRs, but the acceleration timescale must be less than the $\sim 1.5 \mathrm{yr}$ timescale for adiabatic deceleration in the outer heliosphere (Jokipii 1992; Klecker 1995).

It was pointed out by Mewaldt et al. (1996) that the presence of multiply charged ions would help explain the

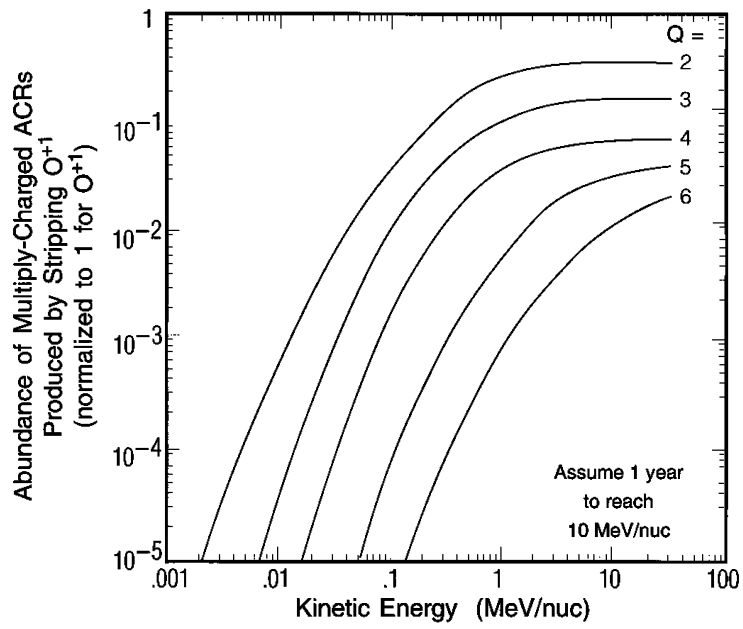

FIG. 4.-Calculated fraction of ACR oxygen with $Q=2-6$ (normalized to 1 for $\mathrm{O}^{+}$) produced by stripping singly charged ACRs during their acceleration. The acceleration time was assumed to be proportional to rigidity, with 1 yr required to reach $10 \mathrm{MeV}$ nucleon $^{-1}$.

acceleration of ACRs to energies greater than $50 \mathrm{MeV}$ nucleon ${ }^{-1}$. According to Jokipii (1990), the "maximum" energy gain $\Delta E$ by an ion with electrical charge $Q e$ at a quasi-perpendicular shock is $\Delta E \approx Q e \Delta \phi$, where $\Delta \phi$ is the electrostatic potential gained in drifting along the shock face. He found $\Delta \phi \approx 240$ MV for the termination shock, corresponding to $\sim 15 \mathrm{MeV}$ nucleon ${ }^{-1}$ for $\mathrm{O}^{+}$, beyond which the $\mathrm{O}^{+}$spectrum should steepen significantly. This implies that singly charged ACRs that lose electrons as they are accelerated will gain additional energy, particularly if they are lost at low energy. The data in Figures 1 and 3 support a picture in which higher charge ions gain more energy, and we regard this as strong support for the diffusive shock-drift acceleration model (Jokipii 1990).

To illustrate the energy spectra of multiply charged ACRs expected from electron stripping, we use a generic shape based on the $\mathrm{O}^{+}$spectrum in Figure 3, extrapolated to lower energies. Cummings et al. (1984) showed that the energy of the peak intensity of various ACR species near $1 \mathrm{AU}$ scaled as $(A / Q)^{-0.91}$ in 1978 while the intensity due to acceleration and solar modulation effects scaled as $(A / Q)^{1.29}$, where they assumed $Q=1$ for ACRs. For source abundances, we use the composition at $3 \mathrm{MeV}$ nucleon ${ }^{-1}$ in Figure 4, an energy that is small compared to the MAST energy range. The resulting spectra, shown in Figure 5, illustrate the expected acceleration of multiply charged ACRs to higher energies. In this example, $56 \%$ of ACRs with energies above $16 \mathrm{MeV}$ nucleon $^{-1}$ are multiply charged, in close agreement with the observations. This empirically based example illustrates how ACRs can attain high energies. It also indicates that electron stripping during acceleration can account for the observed charge state composition with an acceleration rate consistent with theoretical limits on acceleration at the termination shock and with expected rates of energy loss. Jokipii (1996) presents a more complete calculation that includes electron stripping within a self-consistent acceleration and transport model.

The dominance of multiply charged ACRs at high energy can also explain why trapped ACRs have a steeper energy spectrum than interplanetary ACRs (Selesnick et al. 1995) and why the trapped ACR spectrum extends only to $\sim 50 \mathrm{MeV}$ nucleon $^{-1}$, much less than the interplanetary spectrum. To be 


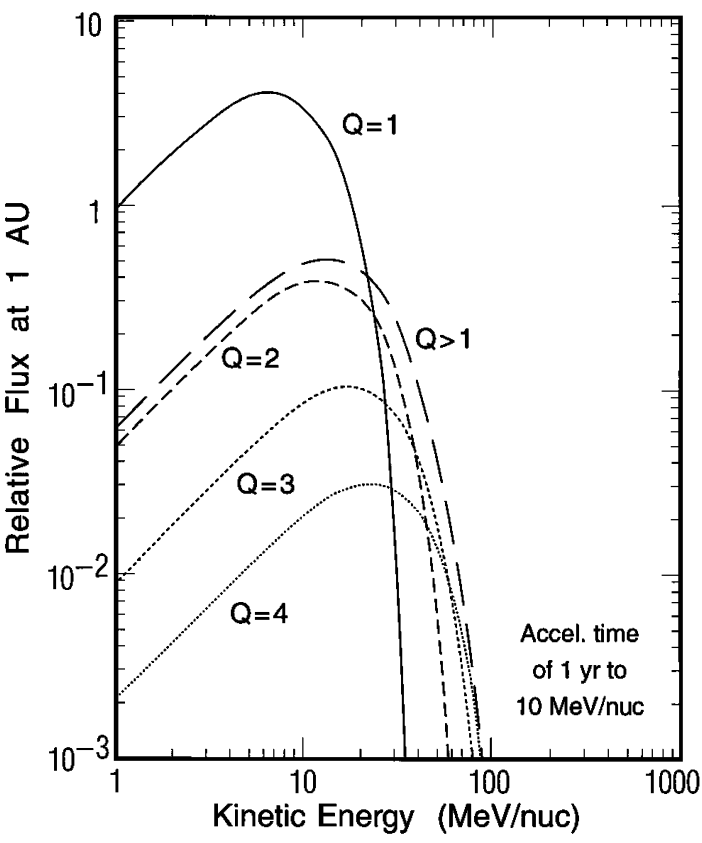

FIG. 5.-Illustration of the energy spectra of ACR oxygen with various charge states that would result from further acceleration of $\mathrm{O}^{+2}, \mathrm{O}^{+3}$, and $\mathrm{O}^{+4}$ produced by stripping $\mathrm{O}^{+}$during its acceleration up to $3 \mathrm{MeV}$ nucleon $^{-1}$ (see Fig. 4). trapped by the Blake \& Friesen (1977) mechanism, interplanetary ions must have access to invariant latitudes below the $\epsilon Q=1$ line in Figure 1 . Since only singly charged oxygen can reach this region, trapped ACRs are derived from only ACR $\mathrm{O}^{+}$and not from multiply charged ACRs that dominate at higher energy.

The results presented here imply that there will also be contributions of multiply charged ACRs to other species $(\mathrm{He}$, $\mathrm{C}, \mathrm{N}, \mathrm{Ne}$, and Ar), which may affect spectral shapes. Indeed, we obtain similar fractions of multiply charged ACRs for high-energy $\mathrm{N}$ and Ne, with lower statistical accuracy. Multiply charged ions should be relatively more abundant in the outer heliosphere because they are affected more by solar modulation processes.

In summary, we find that ACR charge states are much more complex than previously assumed. Calculations indicate that multiply charged ACRs are produced at low energies $\left(<1 \mathrm{MeV}\right.$ nucleon $\left.^{-1}\right)$ during the acceleration process, providing a "clock" that is consistent with a timescale of $\sim 1 \mathrm{yr}$ for acceleration at the termination shock. Multiply charged ACRs will have implications for studies of ACRs throughout the heliosphere.

This work was supported by NASA under grants NAS530704 and NAGW-1919. We appreciate discussions with J. R. Jokipii, B. Klecker, and A. C. Cummings.

\section{REFERENCES}

Adams, J. H. Jr., et al. 1991, ApJ, 375, L45

Blake, J. B., \& Friesen, L. M. 1977, Proc. 15th Int. Cosmic Ray Conf. (Plovdiv, Bulgaria), 2, 341

Boberg, P. R., Tylka, A. J., Adams, J. H., Jr., Beahm, L. P., Fluckiger, E. O.,

Kleis, T., \& Kobel, E. 1995, Adv. Space Res., 17(2), 121

Cummings, A. C., et al. 1995, Geophys. Res. Lett., 22, 341

Cummings, A. C., Stone, E. C., \& Webber, W. R. 1984, ApJ, 287, L99

Fisk, L. A., Kozlovsky, B., \& Ramaty, R. 1974, ApJ, 190, L35

Geiss, J., Gloeckler, G., \& von Steiger, R. 1995, Space Sci. Rev., 72, 49

Hasebe, N., et al. 1994, Geophys. Res. Lett., 21, 3027

Jokipii, J. R., 1990, in Physics of the Outer Heliosphere, ed. S. Grzedzielski \&

D. E. Page (Oxford: Pergamon), 119 1992, ApJ, 393, L41 1996, ApJ, 466, L47

Klecker, B., 1995, Space Sci. Rev., 72, 419

Klecker, B., Hovestadt, D., Gloeckler, G., \& Fan, C. Y. 1980, Geophys. Res. Lett., 7, 1033
Klecker, B., et al. 1995, ApJ, 442, L69

Leske, R. A., Cummings, J. R., Mewaldt, R. A., Stone, E. C., \& von Rosenvinge, T. T. 1995, ApJ, 452, L149

Mewaldt, R. A., et al. 1993, Geophys. Res. Lett., 20, 2263

Mewaldt, R. A., Cummings, J. R., Leske, R. A., Selesnick, R. S., Stone, E., \& von Rosenvinge, T. T. 1996, Geophys. Res. Lett., 23, 617

Oschlies, K., Beaujean, R., \& Enge, W. 1989, ApJ, 345, 776

Palmer, I. D. 1982, Rev. Geophys. Space Phys., 20, 335

Pesses, M. E., Jokipii, J. R., \& Eichler, D. 1981, ApJ, 246, L85

Roederer, J. G. 1970, Dynamics of Geomagnetically Trapped Radiation (New York: Springer)

Selesnick, R. S., Cummings, A. C., Cummings, J. R., Mewaldt, R. A., Stone, E. C., \& von Rosenvinge, T. T. 1995, J. Geophys. Res., 100, 9503

Singh, R. K., Mitra, B., Durgaprasad, N., Biswas, S., Vahia, M. N., Yadav, J. S., Dutta, A., \& Goswami, J. N. 1991, ApJ, 374, 753

Spjeldvik, W. 1979, Space Sci. Rev., 23, 499

Tsyganenko, N. A. 1989, Planet. Space Sci., 37, 5 